\title{
A Miniaturized Reconfigurable CRLH Leaky-Wave Antenna Using Complementary Split-Ring Resonators
}

\author{
Damiano Patron (D), Yuqiao Liu, and Kapil R. Dandekar \\ Department of Electrical and Computer Engineering, Drexel University, 3141 Chestnut Street, Philadelphia, PA 19104, USA \\ Correspondence should be addressed to Damiano Patron; damiano.patron@gmail.com
}

Received 2 June 2017; Revised 12 December 2017; Accepted 11 January 2018; Published 7 March 2018

Academic Editor: John N. Sahalos

Copyright (c) 2018 Damiano Patron et al. This is an open access article distributed under the Creative Commons Attribution License, which permits unrestricted use, distribution, and reproduction in any medium, provided the original work is properly cited.

\begin{abstract}
Composite Right-/Left-Handed (CRLH) Leaky-Wave Antennas (LWAs) are a class of radiating elements characterized by an electronically steerable radiation pattern. The design is comprised of a cascade of CRLH unit cells populated with varactor diodes. By varying the voltage across the varactor diodes, the antenna can steer its directional beam from broadside to backward and forward end-fire directions. In this paper, we discuss the design and experimental analysis of a miniaturized CRLH Leaky-Wave Antenna for the $2.4 \mathrm{GHz}$ WiFi band. The miniaturization is achieved by etching Complementary Split-Ring Resonator (CSRR) underneath each CRLH unit cell. As opposed to the conventional LWA designs, we take advantage of a LWA layout that does not require thin interdigital capacitors; thus we significantly reduce the PCB manufacturing constraints required to achieve size reduction. The experimental results were compared with a nonminiaturized prototype in order to evaluate the differences in impedance and radiation characteristics. The proposed antenna is a significant achievement because it will enable CRLH LWAs to be a viable technology not only for wireless access points, but also potentially for mobile devices.
\end{abstract}

\section{Introduction}

Reconfigurable antennas have received significant attention in the literature with respect to static antennas (antennas with a fixed radiation pattern) thanks to their capability of dynamically changing their radiation properties. These antennas can adapt their characteristics in response to the behavior of the wireless channel and be used for a variety of applications including throughput maximization $[1,2]$, interference management [3], directional networking [4], and security [5].

Adaptive antennas can be divided into two subclasses: phased arrays and reconfigurable antennas. While the former subclass requires multiple radiating elements and phase shifting networks $[6,7]$, the latter subclass of reconfigurable antennas consists of a single radiating element, capable of generating different patterns or polarization $[8,9]$. The reconfigurable antenna solution is thus preferable with respect to a phased array antenna mainly because: (i) it employs a single active element and therefore it occupies a small space and (ii) it allows for high radiation efficiency since it does not employ phase shifters and power dividers.
Various types of reconfigurable antennas capable of changing pattern and polarization have been proposed in the literature. These antennas may employ embedded switches or variable capacitors to change the current distribution on the metallization of the active element $[10,11]$ or may employ an active antenna element surrounded by passive elements (i.e., parasitic elements) loaded with variable capacitors or connected to switches [12,13]. Particularly interesting is the design of Composite Right-/Left-Handed (CRLH) Reconfigurable Leaky-Wave Antennas (LWAs), a two-port metamaterial-based design that is able to steer its directive beam from broadside to backward and forward angles [14].

Leaky-wave antennas are based on the concept of traveling wave, as opposed to conventional resonating-wave behavior. When an RF signal is applied to the input port, the traveling wave progressively "leaks" power as it travels along the waveguide structure. LWAs can also be seen as a phased array traveling wave antenna with amplitude decaying excitation and progressive phase shift as a result of the wave traveling along each unit cell. This leakage phenomenon is directly related to the directivity of the radiated beam. Besides conventional PCB substrates, recent developments in LWA 


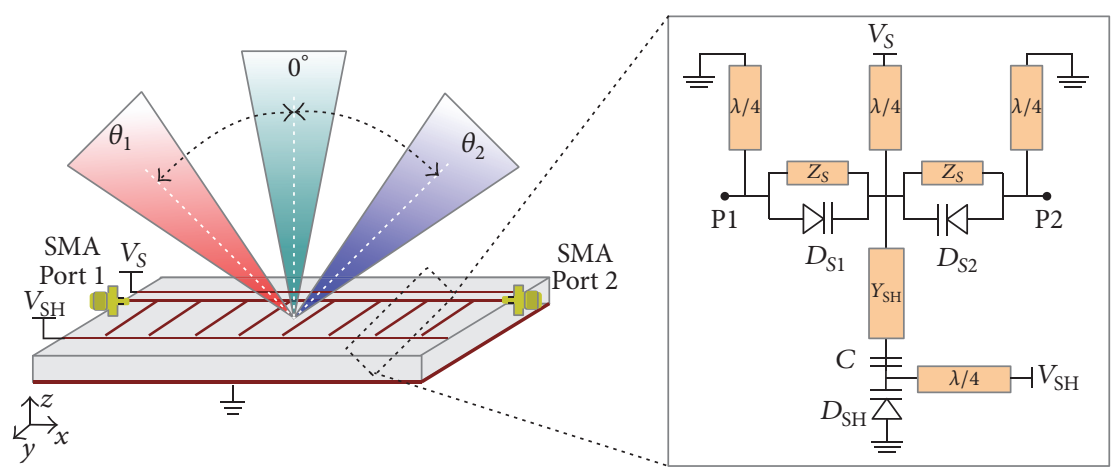

(a) (b)

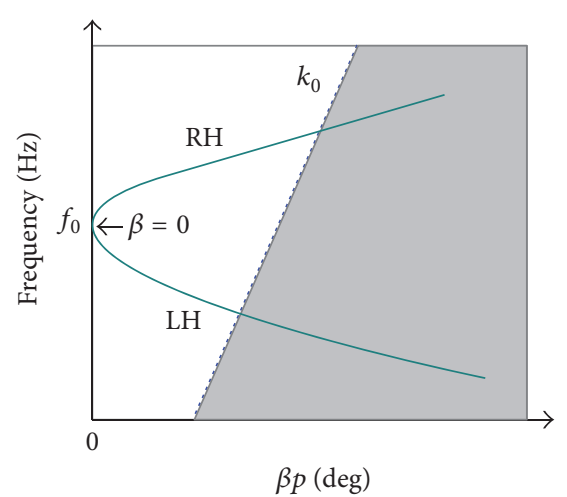

(c)

FIGURE 1: (a) Sketch of a CRLH Leaky-Wave Antenna with beam steering from broadside $\theta=0^{\circ}$ to backward $\theta_{1}$ and forward $\theta_{2}$ angles. (b) Schematic of a conventional CRLH unit cell. (c) Dispersion diagram used to evaluate the propagation constant $\beta$ and estimate the main beam angle $\theta$.

designs have shown the possibility of designing CLRH LWA by using liquid crystals. For example, in $[15,16]$, the authors present CRLH LWA made of injected liquid crystals with the beam steering achieved through an external electric field. As opposed to the proposed LWA, the use of liquid crystals requires very high bias voltage and the beam steering is limited to 20-30 degrees.

Although the planar and compact form factors of the LWAs make them suitable for wireless base stations, they cannot be exploited on mobile devices due to size constrains. In this paper, we will address this limitation by presenting an approach that will make LWAs more suitable for mobile devices.

Current attempts to miniaturize antenna dimensions involve the use of nonconventional substrates with high or enhanced dielectric constant $[17,18]$. Other techniques were developed where the substrate is made by stacking reactive layers $[19,20]$. Unfortunately, these techniques introduce more manufacturing complexity and bulk. In [21], the authors propose the design of a miniaturized CRLH LWA using metallic vias and interdigital lines. Even though the results show a wide beam scanning from -60 to +67 degrees, the beams are characterized by a poor front-to-back ratio and the patterns are not electrically reconfigurable. On the other hand, recent developments in defected ground structures have shown the possibility of properly etching the ground plane of transmission lines or antennas in order to change their cut-off and resonant frequencies $[22,23]$. As a result, devices with small dimension can be loaded with complementary resonators on the ground plane to resonate at lower frequencies, achieving miniaturization [24, 25]. We are unaware of any previous work that has achieved reconfigurable antenna miniaturization through defected ground structures (e.g., designing complementary resonators).

In this paper, we apply a defected ground technique to achieve miniaturization of reconfigurable antennas. In particular, we build upon the LWA design introduced in [26] in which, as opposed to the conventional LWAs, we greatly reduce $\mathrm{PCB}$ manufacturing constraints by avoiding the use of thin interdigital capacitors. In this paper, we designed the miniaturized LWA by applying a CSRR underneath each unit cell to achieve miniaturization of the top layer radiating layout. The unit cell is designed and characterized to resonate at $2.4 \mathrm{GHz}$ and provide the largest possible beam steering. Relative to a conventional $2.4 \mathrm{GHz}$ LWA, the overall dimension can be halved while maintaining good impedance matching, relatively high front-to-back ratio, and good beam steering performance.

The miniaturized LWA is designed to exhibit good impedance matching within the $2.41-2.48 \mathrm{GHz}$ band, for WiFi operations on mobile devices such as laptops or tablets.

The paper is organized as follows: Section 2 provides a brief overview on LWAs and the metamaterial unit cell. Section 3 describes the design of the miniaturized LWA unit cell loaded with CSRR. Section 4 presents the LWA design along with experimental analysis of impedance and radiation patterns. These characteristics are then compared with a nonminiaturized prototype. Finally, conclusions are drawn in Section 5.

\section{Background}

The reconfigurable CRLH LWA can be realized as a 2-port radiating element with tunable radiation properties. The layout is made by a series of $N$ metamaterial unit cells [27], cascaded in order to create a periodic structure from port 1 to port 2, as shown in Figure 1(a). Unlike conventional resonating-wave antennas, the LWA is based on the concept of a traveling wave. When a radio-frequency signal is applied to one of the input ports, the traveling wave leaks out energy as it progressively travels toward the second port. This energy leakage determines the directivity of the radiated beam and is a function of the propagation constant along the structure.

In LWAs, the radiation properties are determined by the complex propagation constant $\gamma=\alpha-j \beta$, where $\alpha$ is the attenuation constant and $\beta$ is the phase constant. While the former corresponds to a loss due to the leakage of energy, the latter determines the radiation angle of the main beam. 


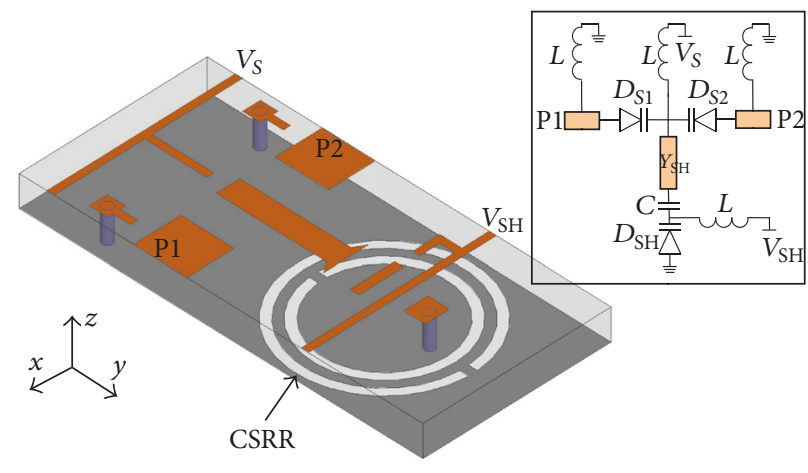

(a)

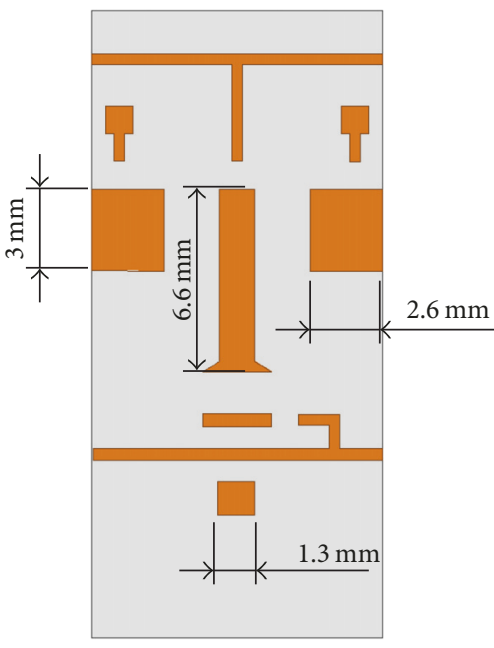

(b)

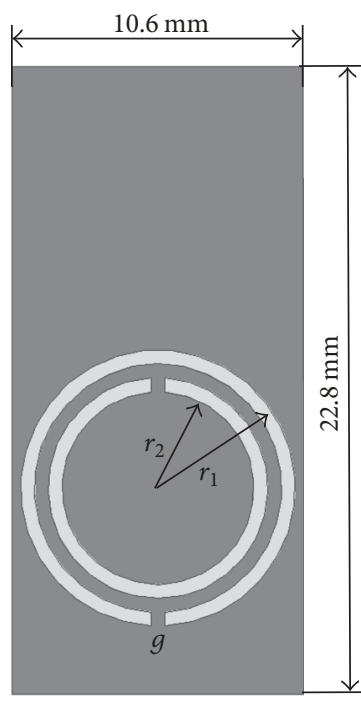

(c)

FIgURE 2: (a) 3D HFSS model of the LWA unit cell, with CSRR etched on the ground plane. (b) 2D top layer layout and dimensions. (c) Bottom layer with ground plane and CSRR design. $r_{1}=5 \mathrm{~mm}$ and $r_{2}=4 \mathrm{~mm}$. The gap $g$ and the distance between the two rings are $0.5 \mathrm{~mm}$.

Additionally, the relationship between $\beta$ and the wavenumber $k_{0}$ defines the regions of operation.

The dispersion diagram in Figure 1(c) depicts the absolute value of $\beta$ and the two regions of operation. The darker area where $|\beta|>k_{0}$ represents the guided wave, where the energy is propagated from port 1 to port 2 , whereas the area where $|\beta|<k_{0}$ represents the radiated region. The angle of the main beam can be determined by the following:

$$
\theta=\sin ^{-1}\left(\frac{\beta}{k_{0}}\right) \text {. }
$$

If we assume that port 2 is fed an input signal and port 1 is terminated with a $50 \Omega$ load, at frequency $f 0$ where $\beta=0$, the antenna radiates a main lobe in broadside direction $\theta=0^{\circ}$, which is perpendicular to the antenna's plane. For frequencies where $\beta>0$ (positive slope of $|\beta|$ ) the antenna operates in $\mathrm{RH}$ region, steering the beam around the left semiplane $\theta_{1}$. On the other hand, when $\beta<0$ (negative slope of $|\beta|$ ) it operates in $\mathrm{LH}$ region, and radiation occurs within the symmetric half-plane $\theta_{2}$. This frequency-dependent behavior allows for the scanning of the main beam from back-fire to end-fire directions. The introduction of tunable capacitances in the unit cell can turn the antenna from a frequency-controlled to a voltage-controlled beam steering radiator.

Several voltage-controlled LWAs have been developed in the literature $[14,28]$ and the circuit model of the conventional metamaterial unit cell can be described as in Figure 1(b). The structure is comprised of both series and shunt components. The series portion is designed with two interdigital capacitors and two varactor diodes $D_{S 1}$ and $D_{S 2}$ connected in parallel. The shunt portion is composed of a stub and a varactor diode $D_{\mathrm{SH}}$ in series. By adding a varactorloaded shunt stub, the shunt admittance $Y_{\mathrm{SH}}$ of the unit cell can also be tuned. In addition, the independent control through $V_{\mathrm{SH}}$ provides an additional degree of freedom, leading to improved tunability of scanning range and impedance matching $[14,28]$. The capacitor $C$ acts as DC-block for the two bias lines $V_{S}$ and $V_{\mathrm{SH}}$. Three $\lambda / 4$ microstrip transformers provide the DC bias lines to the diodes. The introduction of varactor diodes allows for a change in capacitance through the reverse bias voltage, and the propagation constant $\beta$ becomes a function of the diode's voltage. As a result, the curve depicted in Figure 1(c) can be varied along the vertical axis, and the radiator can steer the main beam from backward to forward directions at a given frequency.

Unlike the aforementioned designs, for the miniaturization of the CRLH LWA, we took advantage of an improved design presented in [26] which avoids the use of interdigital capacitors as part of the unit cell model. Therefore, we avoid the manufacturing challenges that may be introduced by etching the very thin fingers that constitute the interdigital capacitors.

From a manufacturing and potential commercialization perspective, this is a significant advantage, especially as research is conducted to miniaturize the layout.

In the next section, we discuss the design of the miniaturized CRLH unit cell along with the CSRR. Through experimental analysis of the scattering parameters ( $S$-parameters) we will evaluate the impedance characteristics and the expected radiation angles from the dispersion diagram.

\section{Design of the CRLH Unit Cell}

The LWA unit cell shown in Figure 2(a) was etched on a conventional FR-4 substrate having dielectric constant $s$ and thickness $t=1.6 \mathrm{~mm}$. The top layout and the CSRR were tuned to operate within the entire $2.4 \mathrm{GHz}$ band.

The unit cell was designed and tuned using the fullwave electromagnetic simulator Ansoft HFSS [29]. In order to 


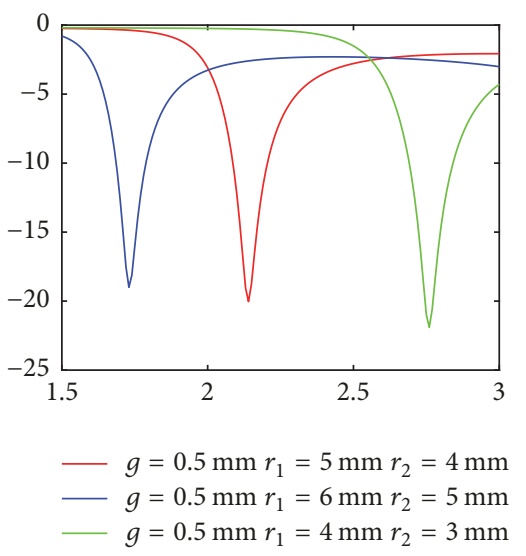

(a)

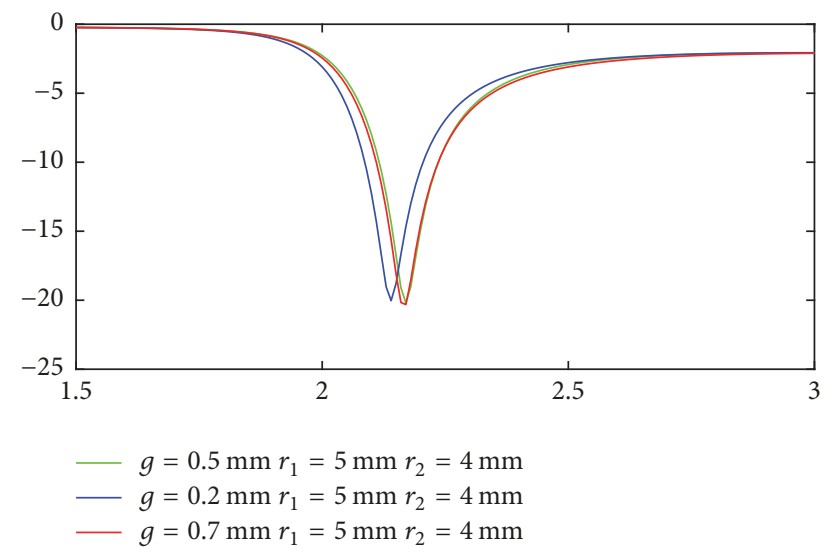

(b)

Figure 3: (a) Parametric simulation of change in inner and outer radii $r_{1}$ and $r_{2}$. (b) Parametric simulation of change in the gap $g$.

perform more realistic simulations, each lumped component was measured through a 2-port fixture and a Vector Network Analyzer (VNA). Then, the $S$-parameters (S2P) were loaded into the circuit simulator Ansoft Designer [30]. The cosimulation between HFSS and Designer allowed for evaluation of the 3D model using the actual S2P parameters. As a varactor diode we selected an Infineon BB833 in SOD323 package, designed to operate up to $2.5 \mathrm{GHz}$ [31]. We chose to use the BB833 because it provided a large dynamic range at low voltages, which reduced the power consumption and the complexity of the control board. In order to get a qualitative evaluation of the capacitance range and loss under reverse bias voltage, we extracted the junction capacitance $C_{J}$ and the series resistance $R_{S}$ from the measured S2P. The plot in Figure 3 shows that the series resistance falls within the range $1.7 \Omega \leq R_{S} \leq 1.85 \Omega$ within the entire reverse voltage sweep. However, when $V_{R} \leq 10 \mathrm{~V}$, the junction capacitance exhibits larger dynamic range: $18 \mathrm{pF} \leq C_{J} \leq 3 \mathrm{pF}$ (Figure 4). The final unit cell layout has been optimized to take advantage of this large $C_{J}$ variation under low bias voltages, achieving the largest possible tunability of the phase constant $\beta$.

The CRLH behavior is determined by designing the unit cell to have proper series capacitance and a shunt inductive component. The series capacitance is achieved by placing two varactor diodes in series with a common cathode $\left(D_{S 1}\right.$ and $D_{S 2}$ ). The inductive part is designed by means of a shunt stub with a varactor diode $\left(D_{\mathrm{SH}}\right)$ placed in series. The dynamic tuning is accomplished by changing the reverse voltage $V_{R}$ of the two bias line $V_{S}$ and $V_{\mathrm{SH}}$. A $C=0.5 \mathrm{pF}$ capacitor was added to the shunt stub in order to decouple the two bias voltages. To further reduce manufacturing complexity and form factor, we have used $L=220 \mathrm{nH}$ inductors that act as RF chokes to provide the two bias voltages. The inset in Figure 2(a) depicts the resulting schematic of the LWA unit cell. The dimensions are shown in Figure 2(b), and the gaps are properly designed to include the lumped components. The series microstrips that connect $D_{S 1}$ and $D_{S 2}$ were calculated and optimized to achieve a characteristic impedance of $50 \Omega$, whereas the shunt microstrip that connect $D_{\mathrm{SH}}$ was scaled down from the reference dimension in [26], due to the

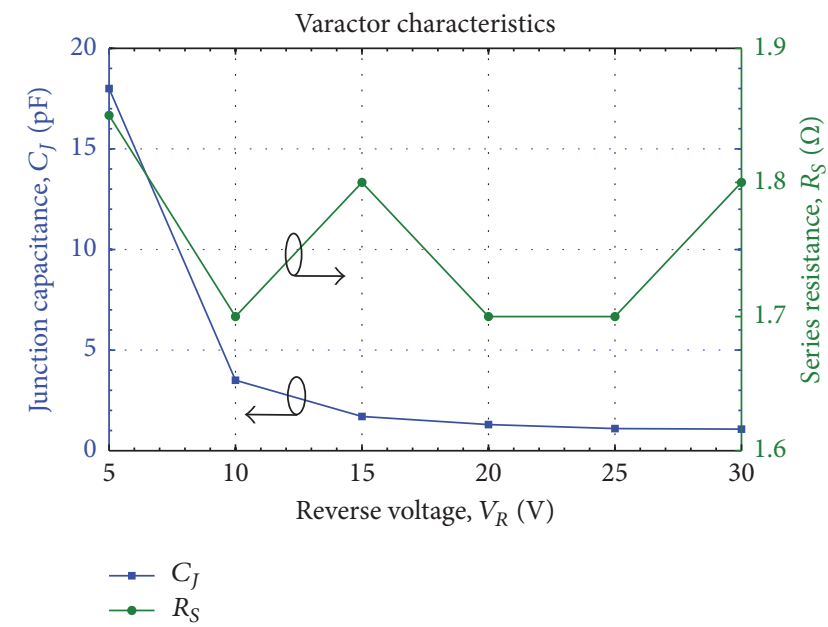

FIGURE 4: Junction capacitance $C_{J}$ and series resistance $R_{S}$ as function of the reverse voltage $V_{R}$. The values were extracted from the measured $S$-parameters. While $R_{S}$ maintains a relatively constant value within the entire voltage sweep, the capacitance $C_{J}$ exhibits a larger dynamic range when $V_{R} \leq 10 \mathrm{~V}$.

contribution of the CSRR. The thinner bias lines that connect the RF chokes were kept as short as possible and the width matches the pads of the SMT chokes.

For lab characterization purpose, the varactor diodes were biased using bench-top power supplies. On the other hand, for real-time dynamic biasing of the LWA, our group developed a control board comprising voltage boost circuits $(0-30 \mathrm{~V})$ and an FPGA. The lookup table contained within the FPGA allows switching between the different pattern configurations and it can be controlled through an external SPI or I2C command.

Simulations have shown that, by using a standard ground plane, the proposed unit cell operates in the frequency region of $5 \mathrm{GHz}$. In order to reduce the operating band, a single CSRR was etched underneath the unit cell. In [32], it has been shown that when a CSRR is etched on the ground plane 

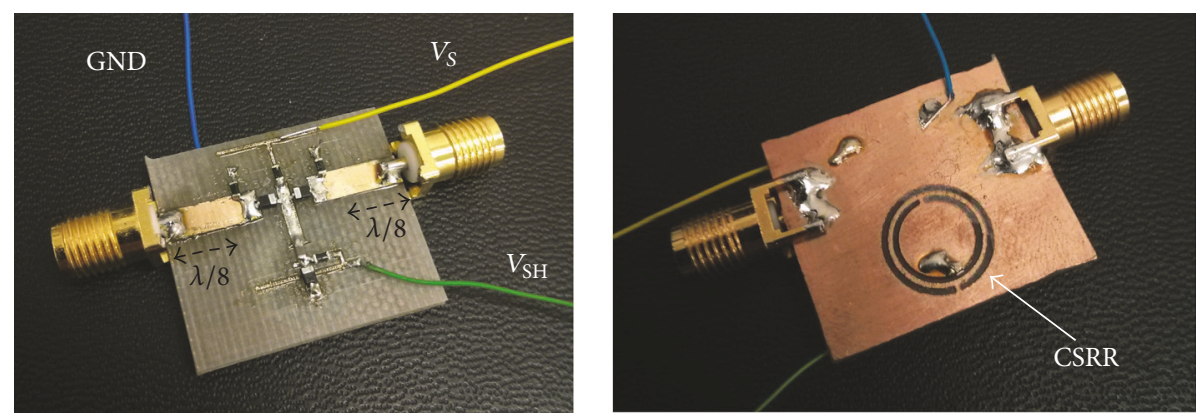

Figure 5: Top and bottom layer pictures of the miniaturized LWA unit cell. The design is etched between two $\lambda / 8$ microstrip lines for $S$ parameter measurements.

of a $50 \Omega$ microstrip line, due to the Babinet principle and, complementarity, the microstrip loaded with CSRR behaves like a one-dimensional effective medium with a negative permittivity within a region around the CSRR resonance. Thanks to this change of permittivity, the top layer antenna structure resonates at lower frequencies.

In order to find the optimal CSRR radii and gap dimensions, we started by simulating the insertion loss of a $50 \Omega$ microstrip line above a parametric CSRR. The simulations have shown a direct proportion between the CSRR radii and the resonant frequency. In fact when the radii are reduced, the resonance shifts down in frequency as shown in Figure 3. The gap $g$ acts as fine-tuning element to optimize the resonance point. In our case, after finding the suboptimal dimension for resonance around $2.4 \mathrm{GHz}$, we choose to fine-tune the CSRR by looking at the frequency reduction of the LWA unit cell down to $2.45 \mathrm{GHz}$. The optimal CSRR layout is shown in Figure 2(c).

The outer radius is $r_{1}=5 \mathrm{~mm}$, while the inner radius is $r_{2}=4 \mathrm{~mm}$. The gap $g$ on both rings, as well as the distance between them, is $0.5 \mathrm{~mm}$. From the simulations, we noticed that when the CSRR is positioned at the center of the unit cell, the miniaturization effect is reduced and the resulting radiation patterns exhibit a pronounced back lobe due to radiation leakage from the CSRR apertures on the ground plane. For this reason, the CSRR was slightly moved from the center to the shunt part of the unit cell, in order to reduce the radiation from the ground plane and enhance the frontto-back ratio. As we will see in Section 3.1, the effects of the CSRR on the unit cell characteristics are to extend the $S_{11}$ bandwidth, while the dispersion curve $\beta p$ is intentionally tuned in the $\mathrm{RH}$ region through the varactor $C_{j}$ operating point and the shunt stub dimension.

The CRLH unit cell can exhibit balanced or unbalanced resonances, based on the series and shunt resonant frequencies $\omega_{\mathrm{se}}, \omega_{\mathrm{sh}}$. While the unbalanced unit cell $\left(\omega_{\mathrm{se}} \neq \omega_{\mathrm{sh}}\right)$ supports two different frequencies, the lower for the LH and the higher for the $\mathrm{RH}$ region, we used balanced unit cell $\left(\omega_{\text {se }}=\omega_{\text {sh }}\right)$ in order to avoid the gap between the RH and LH regions and match the structure over a broad bandwidth. In terms of radiating regions, a CRLH unit cell can typically operate in either $\mathrm{RH}$ or LH regimes. However, in order to achieve the maximum beam coverage by switching between the two input ports, we have optimized the design within the
$\mathrm{RH}$ region $(|\beta|>0)$. Similar to [33], port 1 is used and the beam can be steered from $0^{\circ}$ to $\max \left\{\theta_{2}\right\}$, while by switching to port 2 the beam covers the symmetrical quadrant from $0^{\circ}$ to $\max \left\{\theta_{1}\right\}$. This design choice enables full-space beam steering, while taking advantage of the high $C_{J}$ variation under low voltage regimes. Due to the 2-port switching, a similar beam steering mechanism can be achieved using unbalanced CRLH unit cells.

The next subsection describes the experimental analysis conducted on a miniaturized unit cell prototype. We evaluated the impedance characteristics and the expected radiation angles from the dispersion diagram.

3.1. Characterization Results. S-parameter measurements were carried out to assess the performance of a miniaturized unit cell prototype and validate the simulation results. The unit cell was etched between two $\lambda / 8$ transmission lines for soldering the SMA connectors. An Agilent N5230A Vector Network Analyzer was calibrated with the port extension function for deembedding the two extra lengths. Top and bottom layers of the manufactured unit cell are shown in Figure 5.

Figure 6 shows measured and simulated $S$-parameters for four arbitrary configurations. Due to port symmetry, in this plot, we assume $S_{11}=S_{22}$ and $S_{12}=S_{21}$ for greater visual clarity. By observing the $S_{11}$ curves, we can note that the proposed miniaturized unit cell maintains good impedance matching within the bandwidth of interest from 2.41 to $2.48 \mathrm{GHz}$. The $10 \mathrm{~dB}$ bandwidths are between $220 \mathrm{MHz} \leq$ BW $\leq 650 \mathrm{MHz}$. The measured and simulated $S$-Parameters of the unit cell are in good agreement around the bandwidth of interest: $2.4 \mathrm{GHz}$ and $2.6 \mathrm{GHz}$.

Outside the desired bandwidth, the traces start to differ because of the narrow-band $S$-parameter fixture used for testing. However, we chose to keep a large $x$-axis range in order to highlight the $10 \mathrm{~dB}$ bandwidth.

If we define the phase constant $\beta$ as $\beta=d^{-1} \cos ^{-1}(1+$ $Z(\omega) Y(\omega))$ [27], where $Z(\omega)$ is the series impedance; $Y(\omega)$ is the shunt admittance; the two series varactors $D_{S 1}, D_{S 2}$ vary with $Z(\omega)$ while the shunt varactor $D_{\mathrm{SH}}$ varies with $Y(\omega)$. Furthermore, from our measurements we noticed that the series voltage $V_{S}$ has major control in changing configurations, while the voltage $V_{\mathrm{SH}}$ allows for fine-tuning the $S$-parameters, maintaining the Bloch impedance relatively 

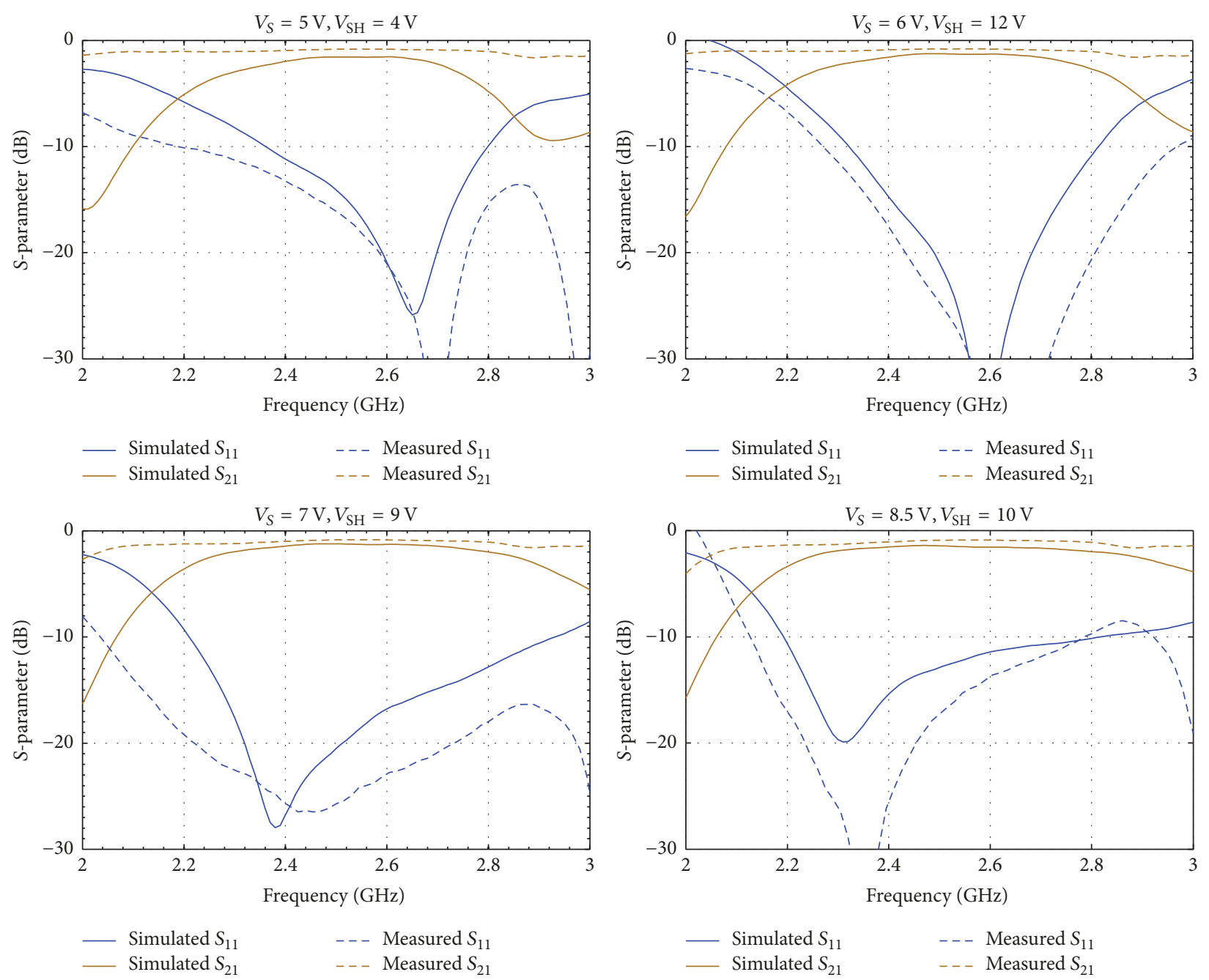

FIGURE 6: Simulated and measured $S$-parameters for four different configurations. While $V_{S}$ acts as major controller for the center frequency, $V_{\mathrm{SH}}$ allows for fine-tuning and improvement of the impedance.

constant and close to $50 \Omega$. The insertion loss, which includes both actual losses and radiation leakage, is between $0.8 \mathrm{~dB} \leq$ $S_{21} \leq 1.5 \mathrm{~dB}$ among the different configurations. The higher deviation between simulation and measurement at the two sides of the bandwidth is potentially due to the $S$-parameters fixture used to extract the S2P of each lumped component.

In order to evaluate the beam steering capabilities, the dispersion diagram was created using the following equation [34] and the measured $S$-parameters:

$$
\beta p=\cos ^{-1}\left(\frac{1-S_{22} S_{22}+S_{21} S_{12}}{2 S_{21}}\right) .
$$

The dispersion diagram in Figure 7 shows the result for four different configurations. We can note that, within the bandwidth of interest, the curves are upward sloping, denoting operation in $\mathrm{RH}$ regime. The expected radiation angles $\theta$ can be estimated through the equation shown in the inset of Figure 7 and computed at the desired frequency. By assuming WiFi operation at $2.46 \mathrm{GHz}$ (channel 11), the miniaturized unit cells allow for steering the radiated beam approximately from $\theta=21^{\circ}$ to $\theta=55^{\circ}$ with respect to

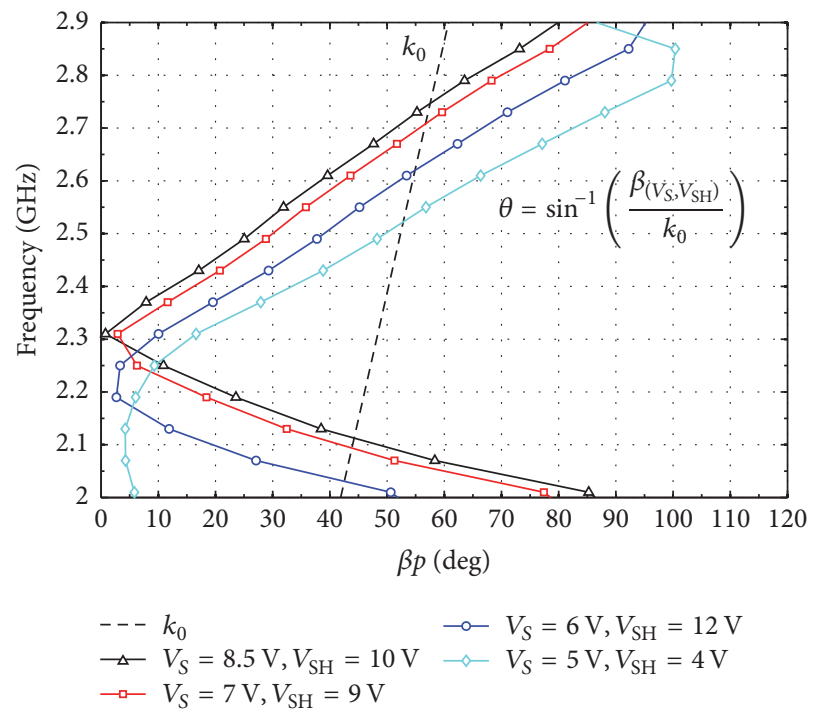

FIGURE 7: Dispersion diagram of the proposed miniaturized CRLH unit cell. The four different states were taken for incremental values of bias voltages $V_{S} V_{\mathrm{SH}}$. The desired frequency bandwidth, 2.41-2.48 GHz, falls within the $\mathrm{RH}$ radiated region. 


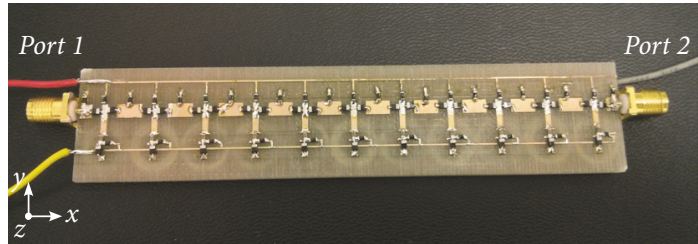

(a)

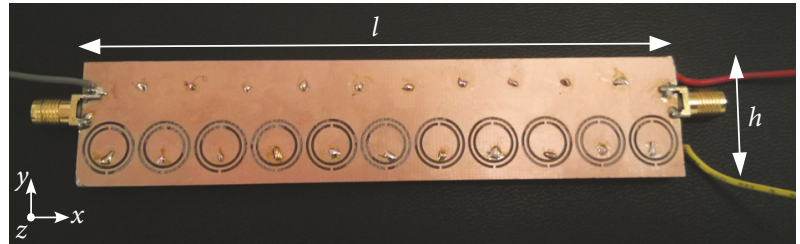

(b)

FIgURE 8: Picture of the miniaturized CRLH LWA. (a) Top layer with cascade or $N=11$ unit cells. (b) Bottom layer with CSRRs. The overall dimensions are $l=11.5 \mathrm{~cm}, h=2.3 \mathrm{~cm}$.
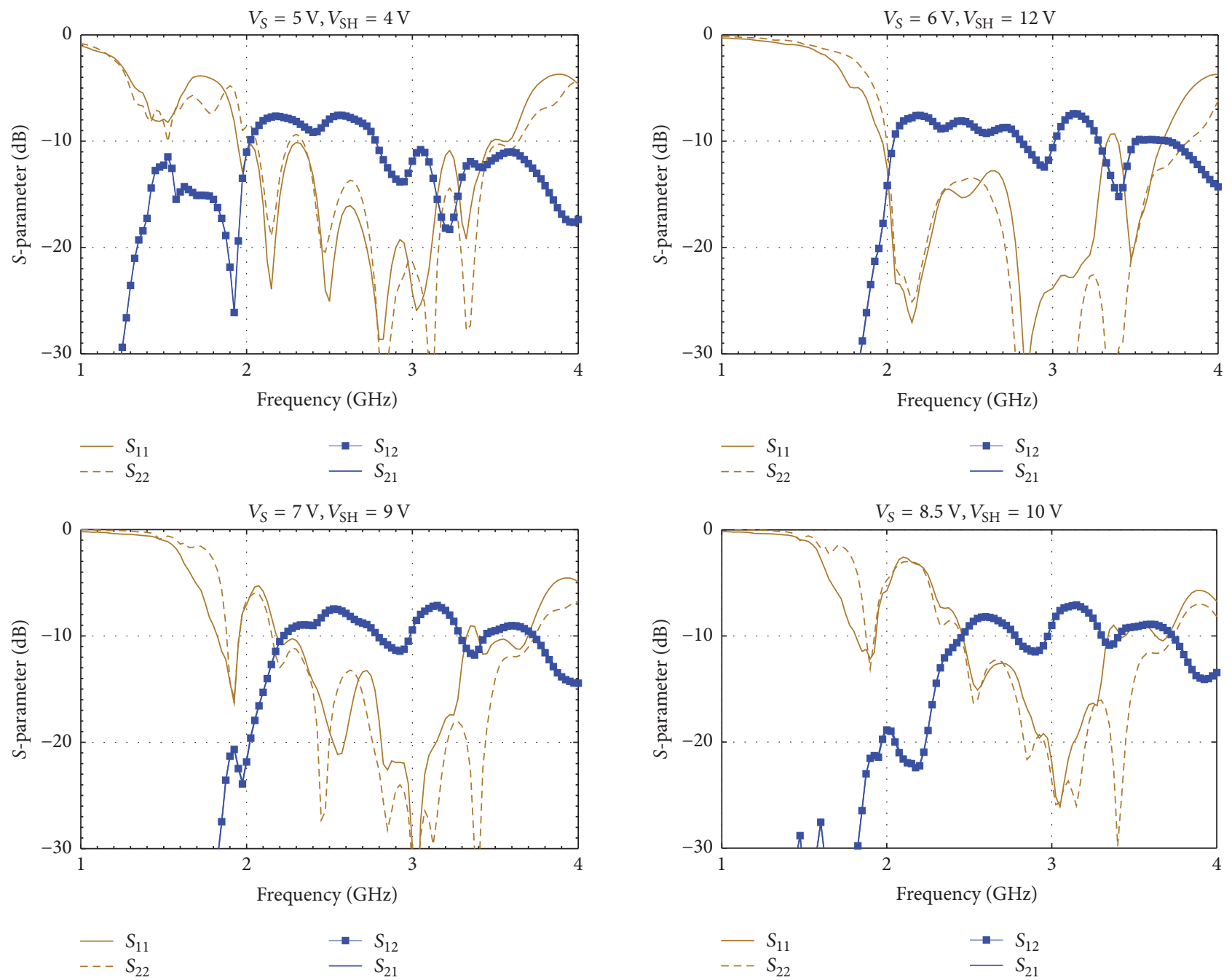

FIGURE 9: Measured S-parameters of the miniaturized CRLH LWA.

broadside direction. The beginning of the flip observed in the $V_{S}=5 \mathrm{~V}, V_{\mathrm{SH}}=4 \mathrm{~V}$ curve is due to the space harmonics periodicity of $\beta$, which is given by

$$
\beta=\beta_{0}+\frac{2 n \pi}{d},
$$

where $\beta_{0}$ is the lowest order mode phase constant, $n$ is the space harmonics $(0, \pm 1, \pm 2, \ldots)$, and $d$ is the period. Although the continuous biasing of varactor diodes allows for a theoretically infinite number of configurations, in
Table 1, we summarize four significant configurations chosen to achieve uniform beam steering based on the HPBW of each beam. The relative Bloch impedance $Z_{b}$ and expected beam angle _ are also reported.

The aforementioned results enable the cascading of the miniaturized unit cell to create a leaky-wave antenna for the $2.4 \mathrm{GHz} \mathrm{WiFi}$ band. In the next section, we discuss the design of this reconfigurable CRLH LWA made by cascading 11 miniaturized unit cells, with experimental analysis of impedance and radiation characteristics. 


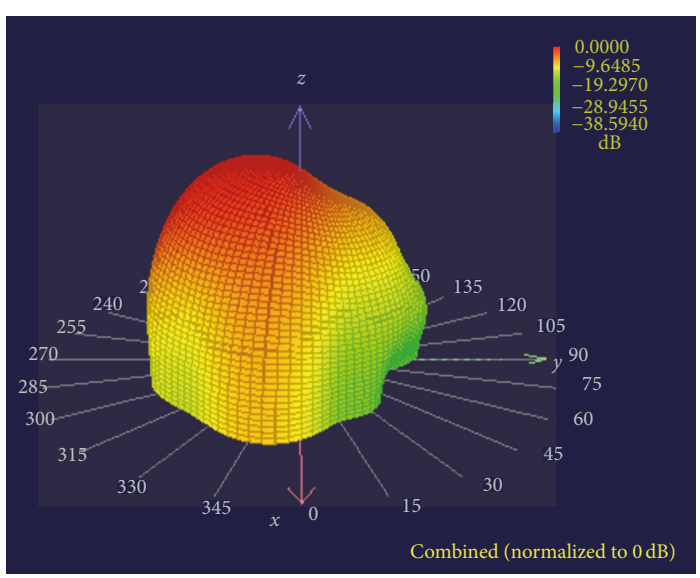

(a)

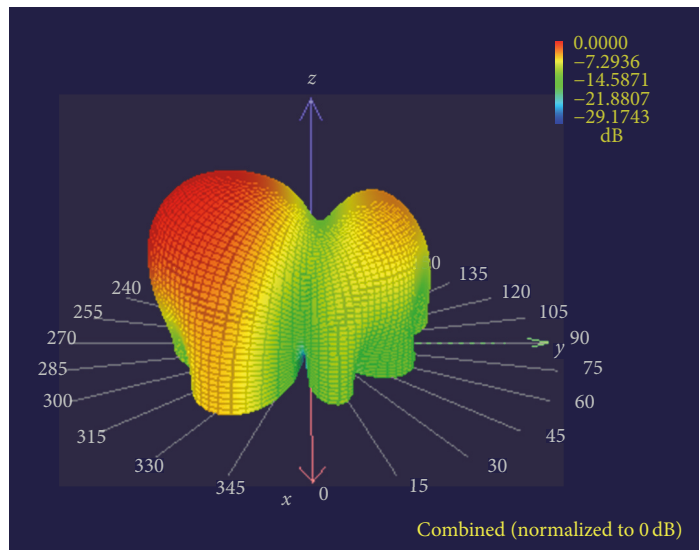

(c)

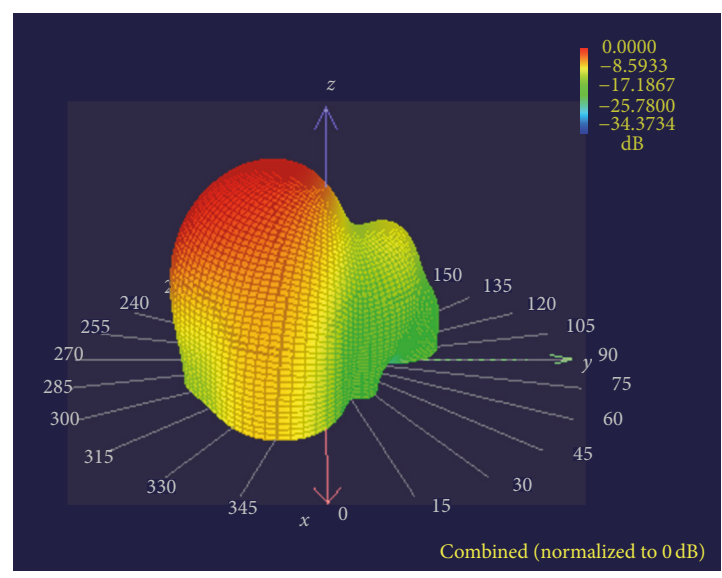

(b)

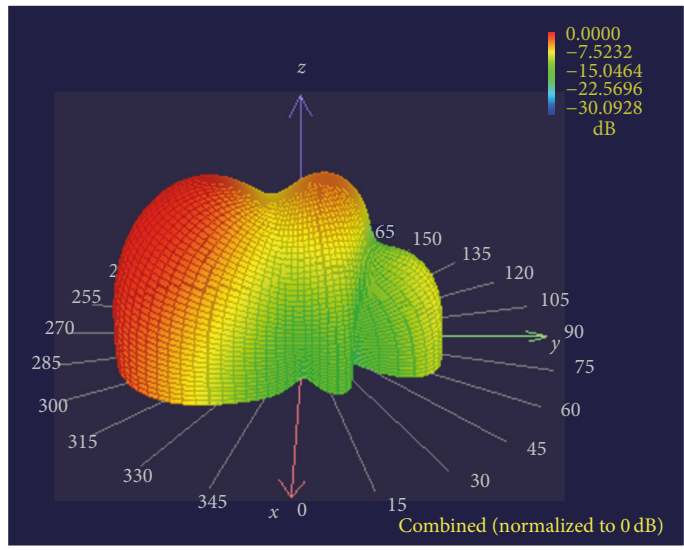

(d)

FIGURE 10: Measured 3D radiation patterns at $2.46 \mathrm{GHz}$ for four configurations. Port 2 oriented in $+y$ direction was connected to a signal generator, while port 1 was terminated to a $50 \Omega$ matched load. (a) $V_{S}=8.5 \mathrm{~V}, V_{\mathrm{SH}}=10 \mathrm{~V}$; (b) $V_{S}=7 \mathrm{~V}, V_{\mathrm{SH}}=9 \mathrm{~V}$; (c) $V_{S}=6 \mathrm{~V}, V_{\mathrm{SH}}=12 \mathrm{~V}$; (d) $V_{S}=5 \mathrm{~V}, V_{\mathrm{SH}}=4 \mathrm{~V}$.

TABLE 1: Summary of four different configurations at the frequency of $2.46 \mathrm{GHz}$

\begin{tabular}{lcc}
\hline Configuration $\left\{V_{S}, V_{\mathrm{SH}}\right\}$ & Block impedance $Z_{b}$ & Beam angle $\theta$ \\
\hline$\{8.5 \mathrm{~V}, 10 \mathrm{~V}\}$ & $42+j 8 \Omega$ & $21^{\circ}$ \\
$\{7 \mathrm{~V}, 9 \mathrm{~V}\}$ & $37+j 7 \Omega$ & $28^{\circ}$ \\
$\{6 \mathrm{~V}, 12 \mathrm{~V}\}$ & $47+j 10 \Omega$ & $38^{\circ}$ \\
$\{5 \mathrm{~V}, 4 \mathrm{~V}\}$ & $56+j 9 \Omega$ & $55^{\circ}$ \\
\hline
\end{tabular}

\section{Miniaturized CRLH Leaky-Wave Antenna}

The periodic structure of the miniaturized CRLH LWA was designed by cascading a series of unit cells described in Section 3. As illustrated in Figure 8, the antenna consists of $N=11$ unit cells and has overall dimension $l=11.5 \mathrm{~cm}$ and $h=2.3 \mathrm{~cm}$. The number of unit cells was selected to achieve positive gain and obtain a fair comparison with the earlier LWA presented in [26]. By switching between the two input ports, the antenna allows for the generation of two independent beams that can be steered from back-fire to end-fire, with expected beam angles $\theta$ estimated during the unit cell analysis.

4.1. Input Impedance. The return loss and the isolation of the two input ports have been measured through a VNA. The $S_{11}$ and $S_{22}$ scattering parameters describe the impedance integrity between the antenna's ports and 50 feedlines, whereas $S_{12}$ and $S_{21}$ render the isolation achievable between them. Figure 9 shows the measured scattering parameters for the four configurations listed in Table 1.

Both input ports exhibit good impedance matching within the $2.41-2.48 \mathrm{GHz}$ band, the small discrepancies between the $S_{11}$ and $S_{22}$ curves are potentially due to the manufacturing process and, in particular, the manual population of the board. We also note that the $10 \mathrm{~dB}$ bandwidth is relatively large, between $1 \mathrm{GHz} \leq \mathrm{BW} \leq 1.3 \mathrm{GHz}$. In terms of decoupling between the two ports, at $2.46 \mathrm{GHz}$, the antenna's isolation is within the range of $8 \mathrm{~dB} \leq S_{21} \leq 10 \mathrm{~dB}$.

4.2. Radiation Pattern. In order to evaluate the radiation characteristics of the proposed antenna and the agreement with the expected angles, we have measured the radiation 


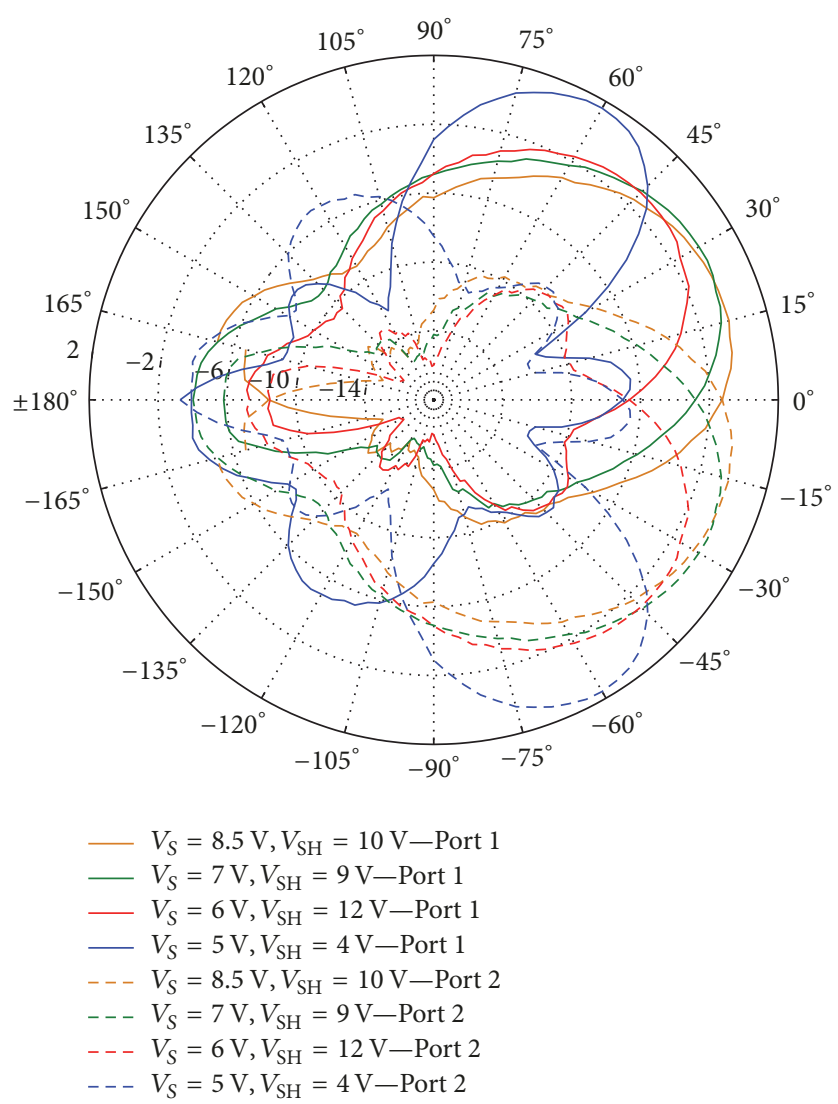

Figure 11: Azimuth $(x-z)$ view of the total beam steering capabilities. The solid lines depict beams generated by exciting port 1 (and port 2 terminated to a $50 \Omega$ load), whereas the dashed lines depict beams generated by exciting port 2 (and port 1 terminated to a $50 \Omega$ load).

patterns for the four configurations listed in Table 1. For this purpose, we used the tool EMSCAN RFxpert [35], which is a bench-top measurement system that enables us to get $3 \mathrm{D}$ and $2 \mathrm{D}$ antenna pattern measurements in real time. Figure 10 shows the 3D antenna directivity graphs measured at $2.46 \mathrm{GHz}$ by exciting port 2 and terminating port 1 to a $50 \Omega$ load. The steering angles are in good agreement with the expected values. The minimum gain is $0 \mathrm{dBi}$ while the peak is about $2 \mathrm{dBi}$, with front-to-back ratio between 5 and $8 \mathrm{~dB}$, depending on the adopted configuration. The front-to-back ratio of the proposed antenna is suboptimal due to the presence of the defected ground plane. In fact a percentage of the total radiation leaks out from the CSRR openings, resulting in some radiation from the back of the PCB. In general, directional antennas that employ CSRR suffer from poor front-to-back ratio [25], but, in the case of the proposed antenna, the patterns maintain a higher level of directivity. The major losses that limit the gain are the series resistance of the varactor diode $R_{S}$ and the lossy FR-4 substrate. More expensive substrates can provide much lower loss factors, while the series resistance of varactor diodes could be improved by choosing a smaller package or a more expensive model. Further measurements were conducted in an anechoic chamber and Figure 11 illustrates the azimuth cut $(x-z)$ with the complete set of radiation patterns accomplished by switching between the two input ports. The total steering angle is about $120^{\circ}$ and the half-power beamwidth
(HPWB) of each beam, between $40^{\circ}$ and $60^{\circ}$, allows for nearly uniform coverage. The measurements in Figure 11 denote good agreement with the expected beam angles listed in Table 1. By comparing the same voltage configurations, the error between the estimated and the measured beam angles is between $0^{\circ}-5^{\circ}$ across all the configurations.

In terms of beams polarization, we observed that the miniaturized CRLH LWA maintains linear polarization across all the configurations, similar to a conventional LWA. In Figure 12 we show the normalized plots of co-pol and cross-pol for four beams at $\pm 60^{\circ}$ and $\pm 30^{\circ}$. For all the configurations, the cross-pol is at least $5 \mathrm{~dB}$ lower than the co-pol confirming that the radiated fields are linearly polarized.

In terms of radiation efficiency, we have estimated that the average components' losses on each LWA unit cell are approximately $0.6 \mathrm{~dB}$ total. Therefore, for the 11-cell LWA presented in this manuscript, the expected radiation efficiency is about $21 \%$ including the ports' return loss.

4.3. Comparison with Conventional LWA. In Figure 13, we compare the size of the proposed miniaturized LWA with an earlier conventional design of a LWA [26]. Both antennas were designed by cascading 11 unit cells; however, the miniaturized LWA is about $53 \%$ smaller than the conventional LWA. We then conducted a qualitative comparison of the electrical and radiation characteristics to evaluate the 

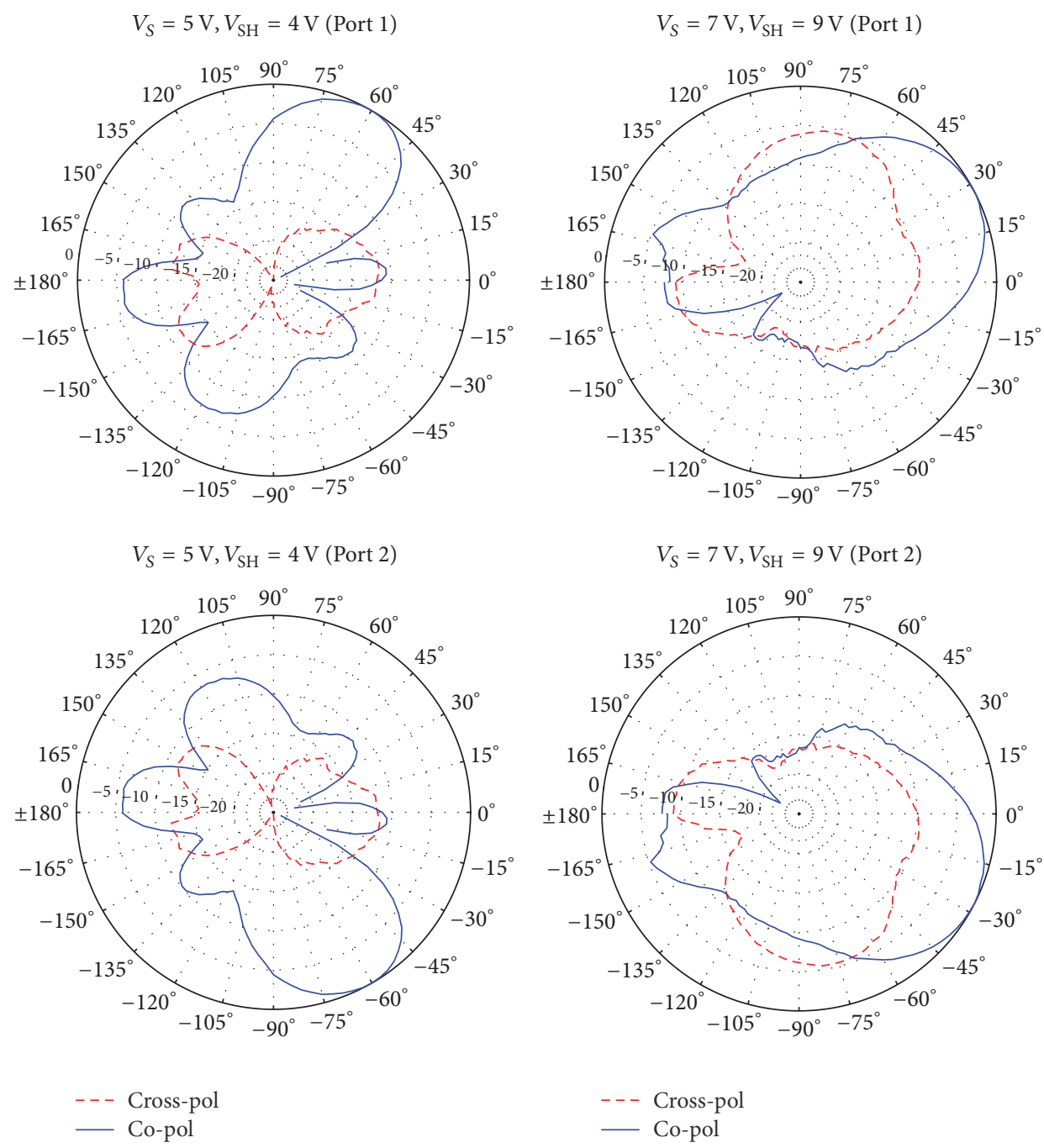

FIGURE 12: Co-pol and cross-pol of the beams at $\pm 60^{\circ}$ and $\pm 30^{\circ}$. The miniaturization of the CRLH LWA maintains the linear polarization, with the cross-pol at least $5 \mathrm{~dB}$ lower than the co-pol.

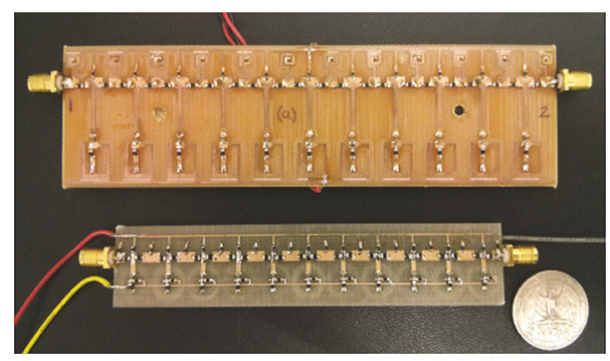

Figure 13: Comparison between the conventional and the proposed miniaturized reconfigurable LWA. Both antennas were designed by cascading $N=11$ unit cells. The former occupies an area of $56 \mathrm{~cm}^{2}$ while the latter occupies an area of $26.5 \mathrm{~cm}^{2}$.

performance of the proposed miniaturized LWA. A summary is shown in Table 2 .

The $10 \mathrm{~dB}$ bandwidth of the miniaturized LWA is significantly larger than the conventional model. However, it is
TABLE 2: Comparison between conventional and miniaturized LWAs.

\begin{tabular}{lcc}
\hline & Conventional LWA & Miniaturized LWA \\
\hline Dimension & $56 \mathrm{~cm}^{2}$ & $26.5 \mathrm{~cm}^{2}$ \\
$10 \mathrm{~dB}$ bandwidth (Max) & $30 \mathrm{MHz}$ & $1.3 \mathrm{GHz}$ \\
Isolation (min) & $10 \mathrm{~dB}$ & $8 \mathrm{~dB}$ \\
Peak gain & $4 \mathrm{dBi}$ & $2 \mathrm{dBi}$ \\
Front-to-back ratio (avg) & $8 \mathrm{~dB}$ & $7 \mathrm{~dB}$ \\
Beam steering coverage & $120^{\circ}$ & $120^{\circ}$ \\
\hline
\end{tabular}

important to recall that, due to the frequency dependency, different frequency regions will exhibit different handedness regions (i.e., $\mathrm{RH}$ or $\mathrm{LH}$ ) and thus different steering angles. Moreover, when the dispersion curve approaches the propagation regime, the beam's directivity, and gain degrade.

Due to the smaller dimension, the isolation between the two input ports is lower with respect to the standard model. Although more than the $85 \%$ of the energy is radiated 
and attenuated through the structure, the employment of a single-pole-double-throw (SPDT) switch would allow further decoupling the two ports and switching between them to generate the desired back-fire and end-fire beams. Furthermore, the cascade of additional unit cells can also lead to higher isolation between the ports and increases the radiated gain.

In terms of radiation characteristics, the miniaturized LWA allows for beam steering of about $120^{\circ}$ around the azimuth plane, similar to the earlier version. The peak gain is $2 \mathrm{~dB}$ lower, but sufficient to utilize the antenna for mobile applications [36]. The front-to-back ratios are comparable, with both antennas performing between 4 and $8 \mathrm{~dB}$, depending on the adopted configuration.

\section{Conclusion}

In this paper, we presented the design of a miniaturized reconfigurable leaky-wave antenna, where the size reduction was accomplished by etching a Complementary Split-Ring Resonator (CSRR) underneath each unit cell.

The CSRR was designed to decrease the size of an improved design of CRLH unit cell, covering the whole WiFi band from $2.41 \mathrm{GHz}$ to $2.48 \mathrm{GHz}$. The absence of interdigital capacitors greatly reduces manufacturing constraints for size reduction while also allowing the application of the CSRR miniaturization technique.

Numerical and experimental analyses of the miniaturized unit cell have shown good impedance performance and relatively large variations of the dispersion curves, which leads to large beam steering.

After fine-tuning the unit cell for the desired radiating region and steering angles, the miniaturized leaky-wave antenna has been designed by cascading 11 unit cells. With respect to an equivalent conventional LWA model, the miniaturized antenna is 53\% smaller and exhibits a larger $10 \mathrm{~dB}$ bandwidth. The radiation patterns were in good agreement with the expected angles, and the total azimuth coverage is about $120^{\circ}$ with gains between 0 and $2 \mathrm{dBi}$.

In conclusion, the technique of etching CSRR on reconfigurable leaky-wave antennas has been shown to be successful for size reduction and maintenance of good radiating performance. The proposed solution enables the development of miniaturized reconfigurable antennas that do not require expensive and customized substrates. In future work, the antenna will be applied on software-defined radios to realize new wireless networking applications exploiting directionality on mobile device platforms.

\section{Conflicts of Interest}

The authors declare that there are no conflicts of interest regarding the publication of this paper.

\section{Acknowledgments}

This material is based upon work supported by the National Science Foundation under Grant CNS-1422964.

\section{References}

[1] J. Kountouriotis, D. Piazza, K. R. Dandekar, M. D’Amico, and C. Guardiani, "Performance analysis of a reconfigurable antenna system for MIMO communications," in Proceedings of the 5th European Conference on Antennas and Propagation, EUCAP 2011, pp. 543-547, ita, April 2011.

[2] N. Gulati and K. R. Dandekar, "Learning state selection for reconfigurable antennas: A multi-armed bandit approach," IEEE Transactions on Antennas and Propagation, vol. 62, no. 3, pp. 1027-1038, 2014.

[3] R. Bahl, N. Gulati, K. R. Dandekar, and D. Jaggard, "Impact of pattern reconfigurable antennas on Interference Alignment over measured channels," in Proceedings of the 2012 IEEE Globecom Workshops, GC Wkshps 2012, pp. 557-562, USA, December 2012.

[4] R. Wang, X. Wang, T. Chow et al., "Capacity and performance analysis for adaptive multi-beam directional networking," in Proceedings of the Military Communications Conference 2006, MILCOM 2006, USA, October 2006.

[5] P. Mookiah, J. Kountouriotis, R. Dorsey, B. Shishkin, and K. R. Dandekar, "Securing wireless links at the physical layer through reconfigurable antennas," in Proceedings of the 2010 IEEE International Symposium on Antennas and Propagation and CNC-USNC/URSI Radio Science Meeting - Leading the Wave, AP-S/URSI 2010, Canada, July 2010.

[6] R. F. Harrington, "Reactively Controlled Directive Arrays," IEEE Transactions on Antennas and Propagation, vol. 26, no. 3, pp. 390-395, 1978.

[7] M. A. Y. Abdalla, K. Phang, and G. V. Eleftheriades, "A planar electronically steerable patch array using tunable PRI/NRI phase shifters," IEEE Transactions on Microwave Theory and Techniques, vol. 57, no. 3, pp. 531-541, 2009.

[8] D. Piazza, P. Mookiah, D. Michele, and K. R. Dandekar, "Pattern and polarization reconfigurable circular patch for MIMO systems," in Proceedings of the 3rd European Conference on Antennas and Propagation, EuCAP 2009, pp. 1047-1051, deu, March 2009.

[9] Y. J. Sung, T. U. Jang, and Y.-S. Kim, "A reconfigurable microstrip antenna for switchable polarization," IEEE Microwave and Wireless Components Letters, vol. 14, no. 11, pp. 534-536, 2004.

[10] D. Patron, K. R. Dandekar, and A. S. Daryoush, “Optical control of reconfigurable antennas and application to a novel patternreconfigurable planar design," IEEE Journal of Lightwave Technology, no. 99, 2014.

[11] Q. Liu and P. S. Hall, "Varactor-loaded left handed loop antenna with reconfigurable radiation patterns," in Proceedings of the 2009 IEEE International Symposium on Antennas and Propagation and USNC/URSI National Radio Science Meeting, APSURSI 2009, USA, June 2009.

[12] D. V. Thiel, "Switched parasitic antennas and controlled reactance parasitic antennas: A systems comparison," in Proceedings of the IEEE Antennas and Propagation Society Symposium 2004 Digest held in Conjunction with: USNC/URSI National Radio Science Meeting, pp. 3211-3214, usa, June 2004.

[13] D. V. Thiel, "Impedance variations in controlled reactance parasitic antennas," in Proceedings of the 2005 IEEE Antennas and Propagation Society International Symposium and USNC/URSI Meeting, pp. 671-674, USA, July 2005.

[14] S. Lim, C. Caloz, and T. Itoh, "Electronically-controlled metamaterial-based transmission line as a continuous-scanning 
leaky-wave antenna," in Proceedings of the 2004 IEEE MITTS International Microwave Symposium Digest, pp. 313-316, usa, June 2004.

[15] B.-J. Che, F.-Y. Meng, J.-H. Fu, K. Zhang, G.-H. Yang, and Q. $\mathrm{Wu}$, "A dual band CRLH leaky wave antenna with electrically steerable beam based on liquid crystals," in Proceedings of the 17th Biennial IEEE Conference on Electromagnetic Field Computation, IEEE CEFC 2016, USA, November 2016.

[16] M. Roig, M. Maasch, C. Damm, and R. Jakoby, "Dynamic beam steering properties of an electrically tuned liquid crystal based CRLH leaky wave antenna," in Proceedings of the 2014 8th International Congress on Advanced Electromagnetic Materials in Microwaves and Optics, METAMATERIALS 2014, pp. 253255, Denmark, August 2014.

[17] J. S. Kula, D. Psychoudakis, W.-J. Liao, C.-C. Chen, J. L. Volakis, and J. W. Halloran, "Patch-antenna miniaturization using recently available ceramic substrates," IEEE Antennas and Propagation Magazine, vol. 48, no. 6, pp. 13-20, 2006.

[18] P. M. T. Ikonen, K. N. Rozanov, A. V. Osipov, P. Alitalo, and S. A. Tretyakov, "Magnetodielectric substrates in antenna miniaturization: Potential and limitations," IEEE Transactions on Antennas and Propagation, vol. 54, no. 11, pp. 3391-3399, 2006.

[19] H. Mosallaei and K. Sarabandi, "Design and modeling of patch antenna printed on magneto-dielectric embedded-circuit metasubstrate," IEEE Transactions on Antennas and Propagation, vol. 55, no. 1, pp. 45-52, 2007.

[20] P. Mookiah and K. R. Dandekar, "Metamaterial-substrate antenna array for MIMO communication system," IEEE Transactions on Antennas and Propagation, vol. 57, no. 10, pp. 32833292, 2009.

[21] G.-C. Wu, G.-M. Wang, H.-X. Peng, X.-J. Gao, and J.-G. Liang, "Design of leaky-wave antenna with wide beam-scanning angle and low cross-polarisation using novel miniaturised composite right/left-handed transmission line," IET Microwaves, Antennas \& Propagation, vol. 10, no. 7, pp. 777-783, 2016.

[22] D. Ahn, J. Park, C. Kim, J. Kim, Y. Qian, and T. Itoh, "A design of the low-pass filter using the novel microstrip defected ground structure," IEEE Transactions on Microwave Theory and Techniques, vol. 49, no. 1, pp. 86-93, 2001.

[23] A. Anand, A. Bansal, K. Shambavi, and Z. C. Alex, "Design and analysis of microstrip line with novel defected ground structure," in Proceedings of the 2013 International Conference on Advanced Nanomaterials and Emerging Engineering Technologies, ICANMEET 2013, pp. 670-673, India, July 2013.

[24] Y. Xie, L. Li, C. Zhu, and C. Liang, "A novel dual-band patch antenna with complementary split ring resonators embedded in the ground plane," in Progress In Electromagnetics Research Letters, vol. 25, pp. 117-126, 2011.

[25] M. S. Sharawi, M. U. Khan, A. B. Numan, and D. N. Aloi, "A CSRR loaded MIMO antenna system for ISM band operation," IEEE Transactions on Antennas and Propagation, vol. 61, no. 8, pp. 4265-4274, 2013.

[26] D. Patron, H. Paaso, A. Mammela, D. Piazza, and K. R. Dandekar, "Improved design of a CRLH leaky-wave antenna and its application for DoA estimation," in Proceedings of the 2013 3rd IEEE-APS Topical Conference on Antennas and Propagation in Wireless Communications, IEEE APWC 2013, pp. 1343-1346, September 2013.

[27] C. Caloz and T. Itoh, Electromagnetic metamaterials transmission line theory and application, John Wiley Sons, Hoboken, NJ, 2006.
[28] D. Piazza, D. Michele, and K. R. Dandekar, "Two port reconfigurable crlh leaky wave antenna with improved impedance matching and beam tuning," in Proceedings of the 3rd European Conference on Antennas and Propagation, EuCAP 2009, pp. 2046-2049, deu, March 2009.

[29] Ansoft HFSS User Manual, Ansoft Corp., Pittsburg, PA, USA, 2005.

[30] Getting Started with Ansoft Designer, Ansoft Corp., Pittsburg, Pa, USA, 2003.

[31] N. Stollon, On-Chip Instrumentation, Springer US, Boston, MA, 2011.

[32] F. Falcone, T. Lopetegi, J. D. Baena, R. Marqués, F. Martín, and M. Sorolla, "Effective negative -/spl epsiv/stopband microstrip lines based on complementary split ring resonators," IEEE Microwave and Wireless Components Letters, vol. 14, no. 6, pp. 280-282, 2004.

[33] H. V. Nguyen, S. Abielmona, and C. Caloz, "Performanceenhanced and symmetric full-space scanning end-switched CRLH LWA," IEEE Antennas and Wireless Propagation Letters, vol. 10, pp. 709-712, 2011.

[34] A. Mehdipour, T. A. Denidni, and A. Sebak, "Multi-band miniaturized antenna loaded by ZOR and CSRR metamaterial structures with monopolar radiation pattern," IEEE Transactions on Antennas and Propagation, vol. 62, no. 2, pp. 555-562, 2014.

[35] “RFxpert User Manual," EMSCAN, 2013, http://www.emscan .com.

[36] K.-L. Wong and P.-J. Ma, "Small-size internal antenna for LTE/WWAN operation in the laptop computer," in Proceedings of the International Conference on Applications of Electromagnetism and Student Innovation Competition Awards, AEM2C 2010, pp. 152-156, Taiwan, August 2010. 


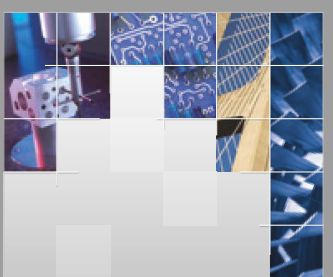

\section{Enfincering}
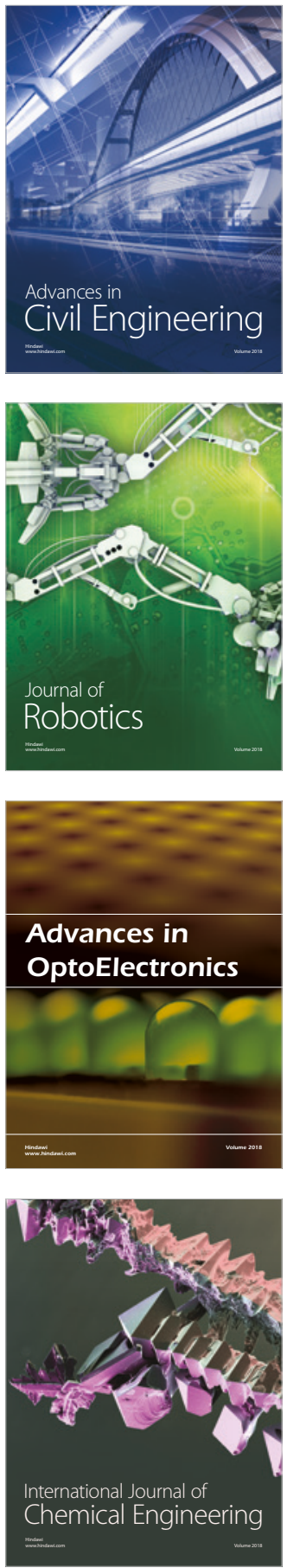

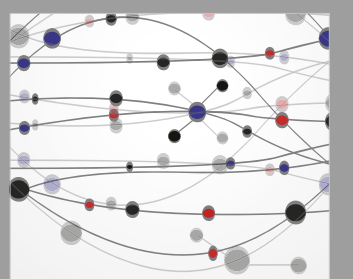

\section{Rotating \\ Machinery}

The Scientific World Journal

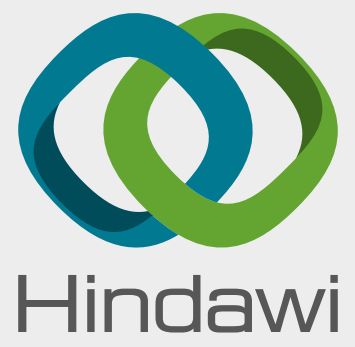

Submit your manuscripts at

www.hindawi.com
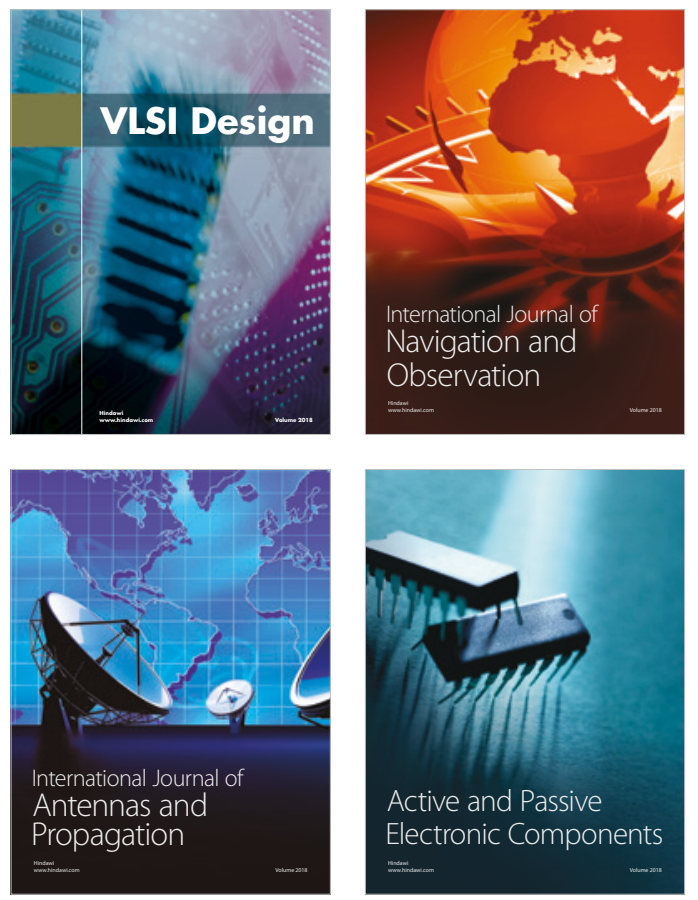
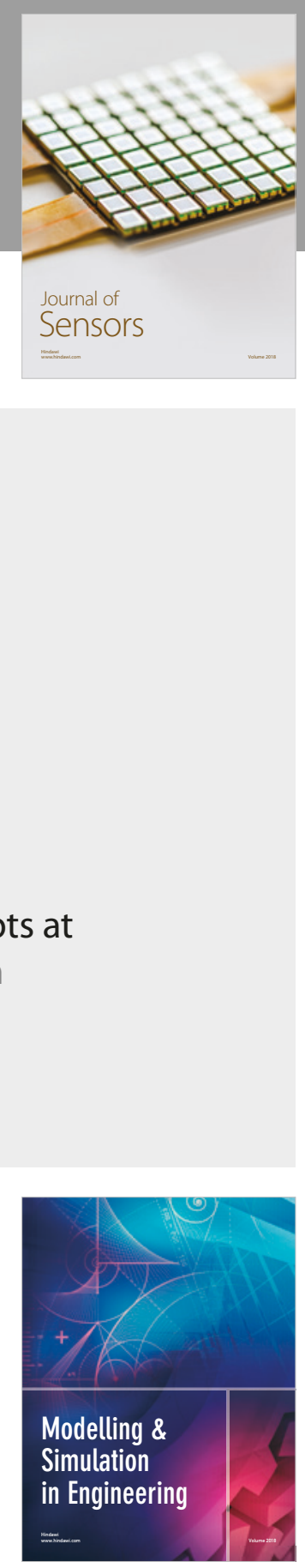

\section{Advances \\ Multimedia}
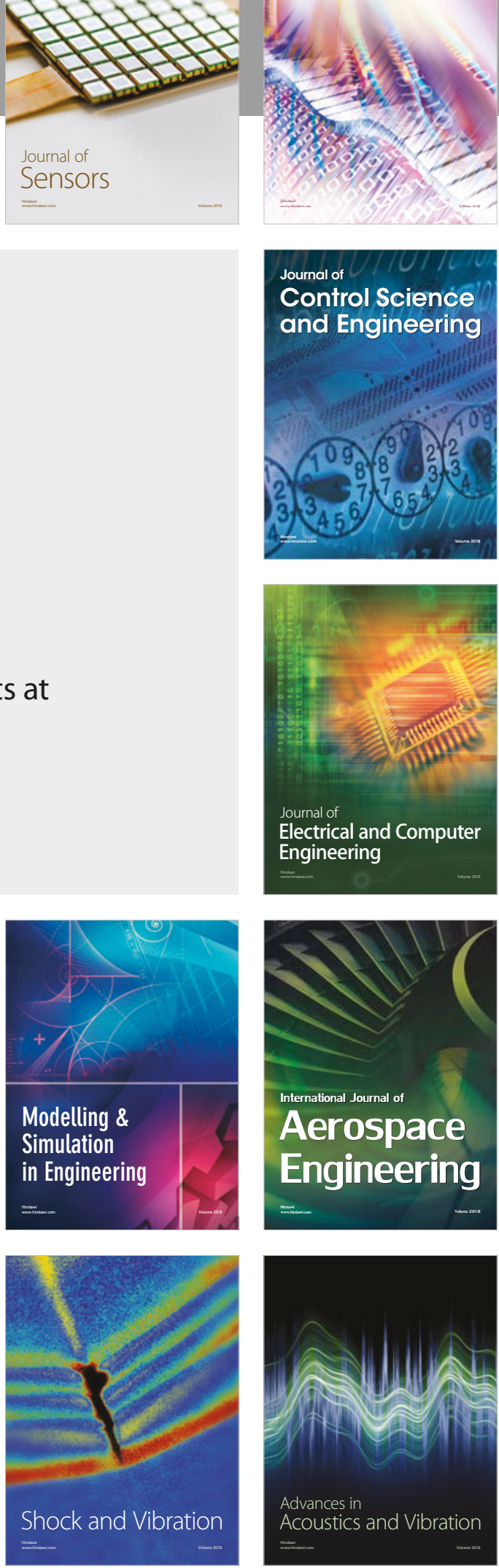